Kazumi Inagaki - Akiko Takatsu - Takuro Watanabe - Yoshie Aoyagi •

Takashi Yarita $\cdot$ Kensaku Okamoto $\cdot$ Koichi Chiba

\title{
Certification of butyltins and phenyltins in marine sediment certified reference material by species-specific isotope-dilution mass spectrometric analysis using synthesized ${ }^{118}$ Sn-enriched organotin compounds
}

Received: 2 June 2006 / Revised: 30 June 2006 / Accepted: 13 July 2006 / Published online: 28 July 2006

(C) Springer-Verlag 2006

\begin{abstract}
A new marine sediment certified reference material, NMIJ CRM 7306-a, for butyltin and phenyltin analysis has been prepared and certified by the National Metrological Institute of Japan at the National Institute of Advanced Industrial Science and Technology (NMIJ/ AIST). Candidate sediment material was collected at a bay near industrial activity in Japan. After air-drying, sieving, and mixing the material was sterilized with $\gamma$-ray irradiation. The material was re-mixed and packaged into 250 glass bottles (15 g each) and these were stored in a freezer at $-30{ }^{\circ} \mathrm{C}$. Certification was performed by use of three different types of species-specific isotope-dilution mass spectrometry (SSID-MS)-SSID-GC-ICP-MS, SSID-GC-MS, and SSID-LC-ICP-MS, with ${ }^{118}$ Sn-enriched organotin compounds synthesized from ${ }^{118} \mathrm{Sn}-$ enriched metal used as a spike. The ${ }^{118}$ Sn-enriched mono-butyltin (MBT), dibutyltin (DBT), and tributyltin (TBT) were synthesized as a mixture whereas the ${ }^{118} \mathrm{Sn}$ enriched di-phenyltin (DPhT) and triphenyltin (TPhT) were synthesized individually. Four different extraction methods, mechanical shaking, ultrasonic, microwaveassisted, and pressurized liquid extraction, were adopted to avoid possible analytical bias caused by non-quantitative extraction and degradation or inter-conversion of analytes in sample preparations. Tropolone was used as chelating agent in all the extraction methods. Certified values are given for TBT $44 \pm 3 \mu \mathrm{g} \mathrm{kg}^{-1}$ as Sn, DBT $51 \pm 2 \mu \mathrm{g} \mathrm{kg}^{-1}$ as Sn, MBT $67 \pm 3 \mu \mathrm{g} \mathrm{kg}^{-1}$ as Sn, TPhT $6.9 \pm 1.2 \mu \mathrm{g} \mathrm{kg}^{-1}$ as $\mathrm{Sn}$, and DPhT $3.4 \pm 1.2 \mu \mathrm{g} \mathrm{kg}^{-1}$ as Sn. These levels are lower than in other sediment CRMs currently available for analysis of organotin compounds.
\end{abstract}

K. Inagaki $(\bowtie) \cdot A$. Takatsu $\cdot$ T. Watanabe $\cdot$ Y. Aoyagi

T. Yarita $\cdot$ K. Okamoto $\cdot$ K. Chiba

Environmental Standard Section, National Metrology Institute of Japan (NMIJ), National Institute of Advanced Industrial Science and Technology (AIST),

Umezono 1-1-1, Tsukuba Central 3,

Tsukuba, Ibaraki 305-8563, Japan

e-mail: k-inagaki@aist.go.jp
Keywords Certification - Certified reference material · Species-specific isotope dilution - Organotin compounds . Sediment

\section{Introduction}

Organotin compounds have wide ranging chemical and toxicological properties. They are widely applied as stabilizers of plastics, as fungicides and pesticides, and as marine antifoulants [1]. Use of tributyltin (TBT) and triphenyltin (TPhT) as marine antifouling agents has led to their almost global dispersal. Although use of TBT and TPhT has been controlled in Japan since 1989, the compounds are still prominent in the coastal sea waters of Japan. Thus, these organotins and their degradation products, di- and mono-organotins, in sediment are frequently monitored to evaluate organotin pollution in water environment. Quantification of organotin species in environmental samples is very difficult, because of their instability and the low concentrations present. Because long-term and worldwide monitoring is necessary for these compounds, comparability of the analytical results is required [2].

The National Metrology Institute of Japan (NMIJ) has already developed, in 2001, a marine sediment certified reference material (NMIJ CRM 7301-a) for analysis of mono, di, and tributyltins (MBT, DBT, and TBT) [3]. Certification was performed using two different types of species-specific isotope-dilution mass spectrometric (SSID-MS) method - gas chromatography coupled with inductively coupled plasma mass spectrometry (SSIDGC-ICP-MS) [4] and SSID-GC-MS combined with a microwave-assisted extraction-in which a mixture of ${ }^{118} \mathrm{Sn}$-enriched butyltins synthesized in our laboratory was used as a spike. In 2005 we developed a new marine sediment certified reference material, NMIJ CRM 7306-a, certified for di and triphenyltins (DPhT and TPhT) and the butyltins by using three different types of SSID-MS method. In the SSID methods, ${ }^{118}$ Sn-enriched TPhT and 
DPhT newly synthesized from ${ }^{118}$ Sn-enriched metal, and a mixture of ${ }^{118} \mathrm{Sn}$-enriched butyltins previously synthesized from ${ }^{118} \mathrm{Sn}$-enriched metal [4] were used as spikes. The certification strategy for NMIJ CRM 7306-a was almost the same as that for NMIJ CRM7301-a [3] but with two improvements to ensure the reliability of the CRM. One was the sterilization of the material with $\gamma$-ray irradiation. NMIJ CRM7301-a was not irradiation-sterilized because of possible degradation of the organotin compounds. Yang et al. reported that significant losses of butyltins in methanol occurs during $\gamma$-ray irradiation whereas such degradation is minimal in a sediment matrix [5]. In our preliminary test, no substantial loss of organotins was observed on $\gamma$-ray irradiation, and thus irradiation-sterilization was performed for CRM7306-a to ensure long term stability. The other improvement was that a total of six combinations of four extraction methods and three SSID-MS methods were used for analysis of the organotin compounds to ensure the reliability of the certified values. The overview of the analysis for certification is shown in Fig. 1. Although SSID-MS methodology can correct most of the systematic errors that occur in the analysis, it can not compensate for non-quantitative extraction from the sample or for species re-conformation in the sample preparation process [6-11]. Indeed, quantitative extraction of MBT is not an easy task, because MBT is strongly adsorbed by sediment matrices, because of its polarity [12-14]. It has been reported that DBT is degraded during microwave-assisted extraction [6] and pressurized liquid extraction [7] when high microwave energy or high extraction temperatures are used, although species interconversion can be taken into account in SSIDMS techniques that furnish data for more than one enriched isotope, as has been described by Encinar and co-workers [6-9]. In this certification, therefore, the four different types of extraction method, mechanical shaking, ultrasonic, microwave-assisted, and pressurized-liquid extraction were adopted to avoid possible analytical biases that caused by non-quantitative extraction and degradation or interconversion of analytes. SSID-liquid chromatography (LC)-ICPMS, was adopted as an SSID-MS method in addition to SSID-GC-ICP-MS and SSID-GC-MS, because organotin separation by LC does not need a derivatization step. Thus, use of SSID-LC-ICP-MS can avoid potential degradation or interconversion of analytes in the derivatization step. This paper mainly describes SSID-MS analysis for certification of NMIJ CRM 7306-a Marine Sediment for organotin compounds.

\section{Experimental}

Preparation of sediment material

The starting material for the CRM was collected in a bay near industrial activity in the Kyushu area, Japan. Approximately $300 \mathrm{~kg}$ surface sediment was sampled and the water was removed by filtration. The sediment material was air-dried for 2 weeks at room temperature $\left(25-28^{\circ} \mathrm{C}\right)$. After removal of visible external materials (rock, shell, etc.) the sediment material was ground in a high-purity alumina ball-mill.
Fig. 1 Overview of analysis for certification

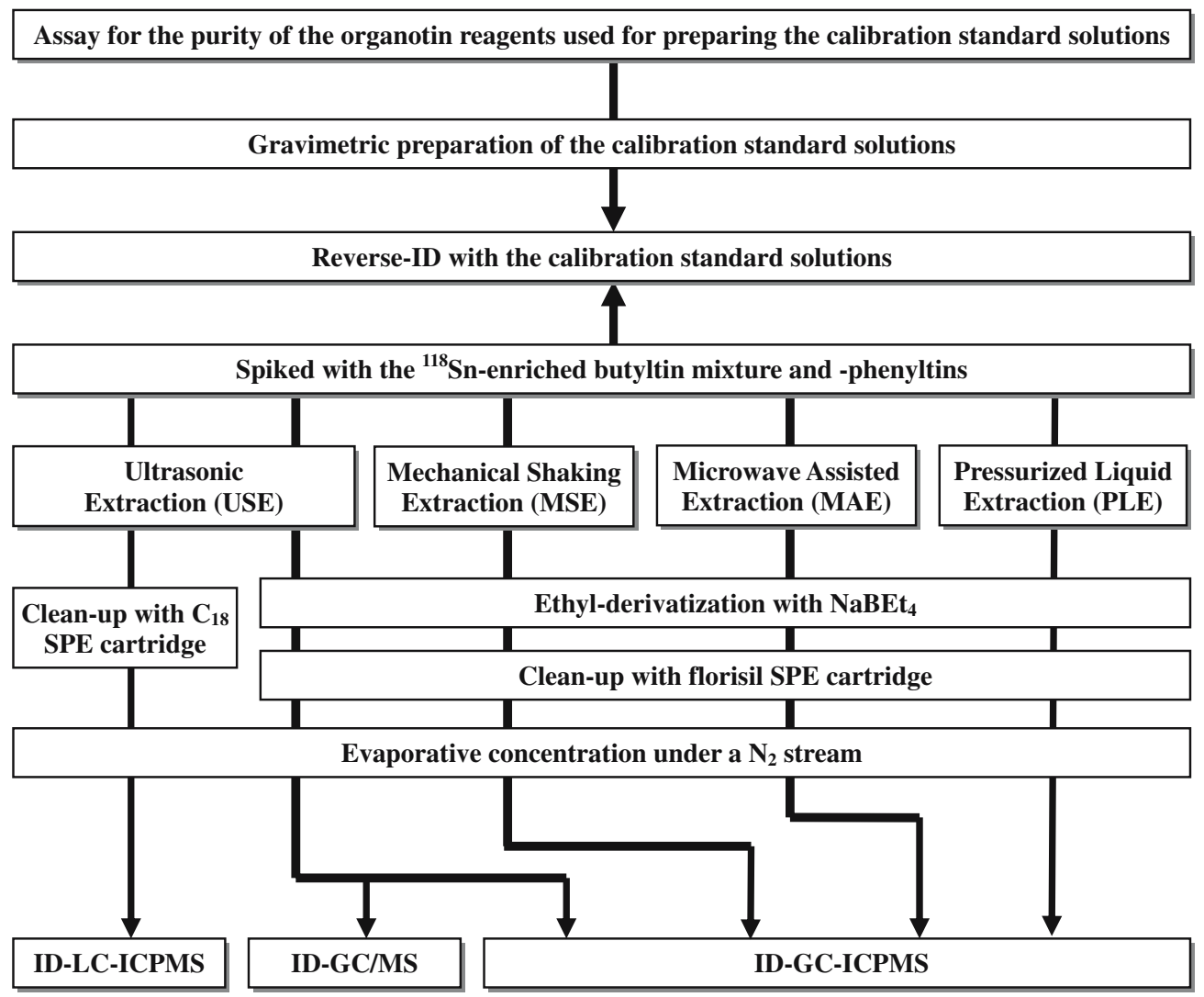


Sediment powder that passed through a $104-\mu \mathrm{m}$ sieve was homogenized using a pan-type mixer. The sediment powder was then bottled in glass bottles $(60 \mathrm{~g}$ each) and irradiation sterilized $\left({ }^{60} \mathrm{Co}, 20 \mathrm{kGy}\right)$. The irradiated powder was re-mixed and homogenized by use of a rocking mixer RM-10S (Aichi Electric, Japan) as a precaution against an inhomogeneous degradation of organotin compounds during sterilization. Finally, the powder was placed in amber glass bottles $(15 \mathrm{~g}$ each) and stored at $-30{ }^{\circ} \mathrm{C}$.

\section{Conversion to dry mass basis}

The concentrations of the constituents of this CRM are given on a dry-mass basis. A dry mass correction factor for sample humidity was determined by drying the sample at $110{ }^{\circ} \mathrm{C}$. After $5 \mathrm{~h}$ the sediment sample reached constant weight, so it was decided the drying time would be $6 \mathrm{~h}$ in this experiment. The dry mass correction factor at the time of certification was $0.959 \pm 0.003$ (average \pm standard deviation for ten different bottles).

\section{Chemicals}

Tributyltin (TBT) chloride, dibutyltin (DBT) chloride, and triphenyltin chloride (TPhT) were purchased from Wako Pure Chemicals (Osaka, Japan). Monobutyltin (MBT) chloride and diphenyltin chloride (DPhT) were purchased from Aldrich (Milwaukee, WI, USA). Tripropyltin (TPrT) chloride was purchased from Merck (Darmstadt, Germany). Standard solutions of each organotin compound except $\mathrm{DPhT}$ were separately prepared by dissolving each compound in methanol (pesticide analysis grade, Wako) to avoid any disproportionation reactions with other organotins. The standard solution of DPhT was prepared by dissolving $\mathrm{DBTCl}_{2}$ in $0.005 \mathrm{~mol} \mathrm{~L}^{-1} \mathrm{HCl}$ in methanol, to prevent selfdisproportionation. All standard solutions were stored at $-20{ }^{\circ} \mathrm{C}$ and diluted working solutions were prepared daily before the analysis. Sodium tetraethylborate $\left(\mathrm{NaBEt}_{4}\right)$ was purchased from Wako Pure Chemicals. A $5 \% \mathrm{~m} / \mathrm{v}$ solution of $\mathrm{NaBEt}_{4}$ was prepared in a glove box that was purged with $\mathrm{N}_{2}$ gas. Other chemicals used were of analytical reagent grade. Pure water prepared by use of a Milli-Q waterpurification system (resistivity $18 \mathrm{M} \Omega \mathrm{cm}$, Nihon Millipore Kogyo, Tokyo, Japan) was used throughout the experiments.

Synthesis of the ${ }^{118} \mathrm{Sn}$-enriched organotin compounds

The mixture of ${ }^{118} \mathrm{Sn}$-enriched butyltin compounds used as the spike for the ID methods were synthesized from ${ }^{118} \mathrm{Sn}$ enriched tin metal $(92 \%$ enriched) purchased from Trace Science International (Ontario, Canada). The synthetic procedure has been described previously [4]. ${ }^{118} \mathrm{Sn}-$ enriched DPhT and TPhT were individually synthesized from ${ }^{118} \mathrm{Sn}$-enriched tin metal $(98 \%$ enriched) purchased from Nippon Sanso (Tokyo, Japan). The synthetic procedures were almost the same as those described elsewhere [15]. A mixture of ca. $0.5 \mathrm{~g}{ }^{118} \mathrm{Sn}$ metal and ca. $2 \mathrm{~g}$ iodine was introduced into a $50-\mathrm{mL}$ round-bottomed flask containing acetic acid $(10 \mathrm{~mL})$ and acetic anhydride $(10 \mathrm{~mL})$. A small crystal of potassium iodide was added as catalyst and the mixture was gently heated to reflux. After cooling in an ice bath, orange crystals of ${ }^{118} \mathrm{SnI}_{4}(1.6 \mathrm{~g})$ were collected. ${ }^{118} \mathrm{SnI}_{4}$ was placed in a 50 -mLroundbottomed flask containing $30 \mathrm{~mL}$ diethyl ether and $1 \mathrm{~mol} \mathrm{~L}^{-1}$ phenyl magnesium bromide in THF was added dropwise. After heating to reflux for $3 \mathrm{~h}$ the white-pink precipitate was collected by filtration after the Grignard reagent had been hydrolyzed with water. The solid was dissolved in dichloromethane and the solution was filtered to remove insoluble impurities. The dichloromethane was removed to leave ${ }^{118} \mathrm{Sn}$-tetraphenyltin (TePhT) as a white solid; this was washed with ethanol. ${ }^{118} \mathrm{SnI}_{4}(0.25 \mathrm{~g})$ and ${ }^{118} \mathrm{Sn}$-TePhT $(0.5 \mathrm{~g})$ were mixed in a glass tube and heated to $200{ }^{\circ} \mathrm{C}$. The reaction products were dissolved in ethanol and the insoluble products were removed by filtration. By the addition of $20 \%$ potassium fluoride solution, ${ }^{118} \mathrm{Sn}$-TPhT fluoride was obtained as an insoluble salt. The fluoride was isolated by filtration, washed with a minimum amount of ethanol, and then treated with conc. hydrochloric acid and extracted with pentane. The extracts were dried with $\mathrm{MgSO}_{4}$ and the pentane was removed to yield ${ }^{118} \mathrm{Sn}$-enriched TPhT chloride as a white powder. ${ }^{118}$ Sn-enriched DPhT dichloride was prepared by treating ${ }^{18} \mathrm{Sn}$-enriched $\mathrm{TePhT}$ with $\mathrm{HCl}$. The product was extracted with pentane. The extracts were dried with $\mathrm{MgSO}_{4}$ and the pentane was removed to yield ${ }^{118} \mathrm{Sn}$-enriched DPhT dichloride.

\section{Extraction procedure}

\section{Ultrasonic extraction}

The ultrasonic extraction procedure for GC-ICP-MS and GC-MS was as follows. The sediment sample (ca. $0.5 \mathrm{~g}$ ) was placed in a PFA centrifuge tube and spiked with an appropriate amount of the spikes. Then $2 \mathrm{~g} \mathrm{NaCl}, 12 \mathrm{~mL}$ toluene containing $0.1 \%$ tropolone, and $10 \mathrm{~mL}$ acetic acidmethanol (1:1) were added to the tubes. The resulting mixtures were extracted in an ultrasonic bath for $30 \mathrm{~min}$ at $60{ }^{\circ} \mathrm{C}$. After addition of $10 \mathrm{~mL}$ water the tubes were again shaken, for good phase separation, and then centrifuged at $3000 \mathrm{rpm}$ for $5 \mathrm{~min}$. Finally, the upper toluene layer was collected as the extract.

For LC-ICP-MS, the extraction solvent was replaced with $10 \mathrm{~mL}$ acetic acid-methanol (1:1) containing $0.1 \%$ tropolone, and the same extraction procedure was performed.

\section{Mechanical shaking extraction}

The sediment sample (ca. $0.5 \mathrm{~g}$ ) was placed in a PFA centrifuge tube and spiked with an appropriate amount of the spikes. Then $2 \mathrm{~g} \mathrm{NaCl}, 12 \mathrm{~mL}$ toluene containing $0.1 \%$ 
tropolone, and $10 \mathrm{~mL} 0.5 \mathrm{~mol} \mathrm{~L}^{-1} \mathrm{HCl}$ in methanol were added to the tubes, and the resulting mixtures were mechanically shaken for $60 \mathrm{~min}$. After addition of $10 \mathrm{~mL}$ water the tubes were again shaken, for good phase separation, and then were centrifuged at $3000 \mathrm{rpm}$ for $5 \mathrm{~min}$. Finally, the upper toluene layer was collected as the extract.

\section{Microwave-assisted extraction}

Closed-vessel microwave-assisted extraction (MAE) was performed. The microwave system used was Mars X (CEM, USA). The sediment sample (ca. $0.5 \mathrm{~g}$ ) was placed in a PFA vessel and spiked with an appropriate amount of the spikes. Then $2 \mathrm{~g} \mathrm{NaCl}, 12 \mathrm{~mL}$ toluene containing $0.1 \%$ tropolone, and $10 \mathrm{~mL} 1 \mathrm{~mol} \mathrm{~L}^{-1}$ acetic acid in methanol were added to the vessel. The microwave irradiation program was ramp to $120{ }^{\circ} \mathrm{C}$ in $10 \mathrm{~min}$ then hold for $10 \mathrm{~min}$. The mixture was transferred to a PFA tube containing $10 \mathrm{~mL}$ water and the tube was shaken, for good phase separation, and then centrifuged at $3000 \mathrm{rpm}$ for $5 \mathrm{~min}$. Finally, the upper toluene layer was collected as the extract.

\section{Pressurized liquid extraction}

Pressurized liquid extraction (PLE) was performed with an ASE 200 instrument (Dionex, USA). The sediment sample (ca. $1 \mathrm{~g}$ ) was placed in an 11-mL stainless extraction cell containing a glass filter and quartz sand and was spiked with an appropriate amount of the spikes. After filling the extraction vessel with quartz sand, the cell was placed in the PLE system. The extraction solvent used was $0.5 \mathrm{~mol} \mathrm{~L}^{-1}$ acetic acid in methanol containing $0.2 \%$ tropolone. The extraction conditions were: oven temp. $110{ }^{\circ} \mathrm{C}$, pressure 10.3 MPa, static time and cycle was $5 \min \times 4$ times. The extract obtained was transferred to a PFA tube containing $10 \mathrm{~mL}$ water and $12 \mathrm{~mL}$ toluene, and the tube was shaken, for good phase separation, and then centrifuged at $3000 \mathrm{rpm}$ for 5 min. Finally, the upper toluene layer was collected as the extract.

\section{Derivatization procedure for $\mathrm{GC}-\mathrm{ICP}-\mathrm{MS}$} and GC-MS

The extracted solutions were transferred to PFA centrifuge tubes and $25 \mathrm{~mL}$ ammonium-acetate buffer $\left(0.5 \mathrm{~mol} \mathrm{~L}^{-1}\right.$,
$\mathrm{pH} 5$ ) and $0.2 \mathrm{~mL} 5 \% \mathrm{NaBEt}_{4}$ solution were added. The tubes were mechanically shaken for $20 \mathrm{~min}$, for ethylation and extraction, and then centrifuged to achieve phase separation. The toluene layers were also transferred to the tubes and mixed with $2 \mathrm{~g}$ anhydrous sodium sulfate to remove the water.

\section{Clean-up procedure}

Clean-up on a Presep-C Florisil cartridge (Wako, Japan) was performed after the derivatization, except for LC-ICPMS measurement. The toluene layer was evaporated to ca. $2 \mathrm{~mL}$ under a stream of $\mathrm{N}_{2}$ gas and loaded on to a cartridge previously conditioned with hexane. The eluent from the cartridge was collected in a $15-\mathrm{mL}$ glass centrifuge tube. Hexane $(6 \mathrm{~mL})$ containing $5 \%(v / v)$ diethyl ether was then also loaded on the cartidge and the eluent was collected in the glass tube. Finally, the collected eluent was evaporated to $0.2 \mathrm{~mL}$ under a stream of $\mathrm{N}_{2}$ gas and used as the measurement sample solution.

For the LC-ICP-MS measurement, a clean-up procedure using Presep-C18 cartridge (Varian, Australia) was performed. The extract was loaded on to a cartridge previously conditioned with acetone. The eluent from the cartridge was collected in a $15-\mathrm{mL}$ glass centrifuge tube, evaporated to $0.2 \mathrm{~mL}$ under a stream of $\mathrm{N}_{2}$ gas, and used as the measurement sample solution.

\section{Determination of organotin compounds} by ID-GC-ICP-MS

The GC-ICP-MS used was that the GC (Agilent 6890GC) was coupled with an ICP-MS (HP4500, Yokogawa Analytical Systems, Tokyo, Japan) by means of a manufactured transfer-line (Yokogawa Analytical Systems). The GC column was HP-1 $\mathrm{ms}(30 \mathrm{~m} \times 0.32 \mathrm{~mm}$ i.d., $0.32 \mu \mathrm{m}$ film thickness). The measured masses were $m / z 118$ and 120. The operating conditions and procedures for GC-ICPMS were similar to those described elsewhere [4].

The concentrations of organotin compounds were calculated by inserting each value into the ID equation Eq. (1), based on a double-ID method [16, 17]:

$$
C_{\mathrm{x}}=\left[\frac{P \cdot D}{M w} \cdot E \cdot \frac{m_{\mathrm{y}}}{w \times m_{\mathrm{x}}} \cdot \frac{m_{\mathrm{z}}}{m_{\mathrm{y}}^{\prime}} \cdot \frac{K_{\mathrm{y}} \cdot R_{\mathrm{y}}-\sum(K \cdot R) / n}{\sum(K \cdot R) / n-R_{\mathrm{x}}} \cdot \frac{\sum\left(K^{\prime} \cdot R \prime\right) / n-R_{\mathrm{z}}}{K_{\mathrm{y}} \cdot R_{\mathrm{y}}-\sum\left(K^{\prime} \cdot R^{\prime}\right) / n}\right]-B
$$

where $C_{\mathrm{x}}$ is the analyte concentration in the sample $\left(\mathrm{mol} \mathrm{g}^{-1}\right), m_{\mathrm{x}}$ the mass of sample (g) used for the samplespike mixture, $m_{\mathrm{y}}$ the mass of spike solution $(\mathrm{g})$ used for the sample-spike mixture, $m_{y}^{\prime}$ the mass of spike solution $(\mathrm{g})$ used for the standard-spike mixture, $m_{\mathrm{z}}$ the mass of standard solution (g) used for standard-spike mixture; $R$ is 
the ${ }^{120} \mathrm{Sn} /{ }^{118} \mathrm{Sn}$ ratio in the sample-spike mixed solution, $R^{\prime}$ is the ${ }^{120} \mathrm{Sn} /{ }^{118} \mathrm{Sn}$ ratio in the standard-spike mixed solution, $R_{\mathrm{x}}$ is the ${ }^{120} \mathrm{Sn} /{ }^{118} \mathrm{Sn}$ ratio in the sample solution, $R_{\mathrm{y}}$ is the ${ }^{120} \mathrm{Sn} /{ }^{118} \mathrm{Sn}$ ratio from the spike solution, $R_{\mathrm{z}}$ is the ${ }^{120} \mathrm{Sn} /{ }^{118} \mathrm{Sn}$ ratio in the standard solution, $w$ is the correction factor for dry mass; $n$ is the number of replicate measurements, and $k, K_{\mathrm{y}}$, and $K^{\prime}$ are the mass discrimination correction factors for each isotope ratio, which were calculated from the area ratio of ${ }^{120} \mathrm{Sn} /{ }^{118} \mathrm{Sn}$ for TPrT in each chromatographic run [4]. $P$ is the purity of each organotin chloride reagent used for preparing the standard solutions, $D$ is the dilution factor for each organotin chloride in the gravimetric dilution of the standard solutions, $M w$ the molecular weight of each organotin chloride, $B$ the procedure blank, and $E$ the variation introduced by extraction to the analytical results.

\section{Determination of organotins by ID-GC-MS}

The GC-MS used was Agilent model 6890GC/5983MSD (Agilent Technologies, Wilmington, DE, USA) with an HP-5 ms column $(30 \mathrm{~m} \times 0.32 \mathrm{~mm}$ i.d., $0.25 \mu \mathrm{m}$ film thickness). The measured masses were $m / z 231$ and 233 for MBT, $m / z 261$ and 263 for DBT and TBT, $m / z 301$ and 303 for DPhT, and $\mathrm{m} / \mathrm{z} 349$ and 351 for TPhT. The other operating conditions and procedures for GC-ICP-MS analysis were similar to those described previously [3].

The concentrations of organotins were calculated by inserting each value into Eq. (2), below, in which it was assumed that the relationship between mass ratio and peak ratio was linear.

$$
C_{\mathrm{x}}=\frac{P \cdot D}{M w} \cdot E \cdot \frac{m_{\mathrm{y}}}{w \cdot m_{\mathrm{x}}\left[\left(R-R_{\mathrm{L}}\right)\left(R W_{\mathrm{H}}-R W_{\mathrm{L}}\right) /\left(R_{\mathrm{H}}-R_{\mathrm{L}}\right)+R W_{\mathrm{L}}\right]-B}
$$

where $C_{\mathrm{x}}$ is the analyte concentration in the sample $\left(\mathrm{mol} \mathrm{g}^{-1}\right), m_{\mathrm{x}}$ the mass of sample ( $\mathrm{g}$ ) used for the samplespike mixture, $m_{\mathrm{y}}$ the mass of spike solution $(\mathrm{g})$ used for the sample-spike mixture, $R W_{\mathrm{L}}$ the ratio of the mass in the spike solution $(\mathrm{g})$ to the mass in the standard solution (lower level), $R W_{\mathrm{H}}$ the ratio of mass in the spike solution (g) to the mass in the standard solution (higher level), $R$ the measured abundance ratio in the sample-spike mixed solution, $R_{\mathrm{L}}$ the measured abundance ratio in the standardspike mixed solution (lower level), $R_{\mathrm{H}}$ the measured abundance ratio in the standard-spike mixed solution (higher level), $w$ the correction factor for dry mass, $P$ the purity of each organotin chloride reagent used for the preparing the standard solutions, $D$ the dilution factor for each organotin chloride in gravimetric dilution of the standard solutions, $M w$ the molecular weight of each organotin chloride, $B$ the procedure blank, and $E$ the variation introduced by the extraction to the analytical results. The mass bias correction was not performed for GC-MS measurements. In this experiment we prepared several sample-spike mixed solutions of different mass ratios and chose the solutions having values of $R_{\mathrm{L}}$ and $R_{\mathrm{H}}$ slightly lower and higher than the $R$ value for calculation.

Determination of organotin compounds by ID-LC-ICP-MS

The LC-ICP-MS system used consisted of a PU-712i HPLC pump (Inert model; GL Science, Tokyo, Japan), a Nanospace SI-2 auto-injection sampler (Siseido, Tokyo, Japan), and an Agilent 7500c ICP-MS (Yokogawa
Analytical Systems, Japan). The LC column used was a Mitysil RP-18 GP $(150 \mathrm{~mm} \times 2.0 \mathrm{~mm}$ i.d. $3 \mu \mathrm{m})$. All tubing used was $1 / 16$ in $(0.13 \mathrm{~mm}$ i.d.) PEEK. The mobile phase was acetonitrile-water-acetic acid-tetraethylammonium chloride-tropolone 65:30:5:0.1:0.075 $(\%, v / v)$. The mobile phase flow rate was $200 \mu \mathrm{L} \mathrm{min}^{-1}$ and the sample volume injected was $5 \mu \mathrm{L}$. The sample-introduction device for ICP-MS was modified from the default settings as follows: a PFA $\mu$ flow of $50 \mu \mathrm{L} \mathrm{min}{ }^{-1}$ was used as a nebulizer, the double-path Scott type spray chamber was cooled to $-5{ }^{\circ} \mathrm{C}$, a $1.0 \mathrm{~mm}$ dimmer injector torch was used, and additional $\mathrm{O}_{2}\left(0.1 \mathrm{~L} \mathrm{~min}^{-1}\right)$ gas was mixed with the argon axial gas flow via a T-adaptor. The LC column was directly connected via a 1/16 PEEK adaptor. The concentrations of organotins were calculated by use of Eq. (1).

\section{Homogeneity study}

The between-bottle homogeneity of the CRM was determined by analyzing sub-samples taken from ten bottles selected from the lot of 250 bottles. All the organotin compounds were determined by ID-GC-ICPMS after ultrasonic extraction. Analysis of variance (ANOVA) of the data was performed and mean squares within group $\left(M S_{\text {within }}\right)$ and among group $\left(M S_{\text {among }}\right)$ were calculated. Then standard deviations between bottles $\left(s_{\mathrm{bb}}\right)$ were calculated by use of Eq. (3):

$s_{b b}=\sqrt{\frac{M S_{\text {among }}-M S_{\text {within }}}{n}}$ 
If the measurement method was insufficient repeatable, the effect of analytical variation on the standard deviation between units $u_{\mathrm{bb}}$ was calculated and used to estimate the inhomogeneity [18]. The $u_{\mathrm{bb}}$ was calculated by use of Eq. (4):

$u_{b b}=\sqrt{\frac{M S_{\text {within }}}{n}} \sqrt[4]{\frac{2}{\nu_{M S_{\text {within }}}}}$

where $\nu_{M S_{\text {within }}}$ denotes the degrees of freedom of $M S_{\text {within }}$.

\section{Results and discussion}

Homogeneity study

In the homogeneity study, between-bottle inhomogeneity $\left(s_{\mathrm{bb}}\right)$ was only observed for TPhT, so $u_{\mathrm{bb}}$ was used as uncertainty-derived inhomogeneity for TPhT. A relatively large $s_{\mathrm{bb}}$ value $(7.1 \%)$ was obtained for DPhT, although this value was not very large compared with uncertainties from other sources, for example the uncertainty of analytical results and between-method variance, as is shown later. These results indicate that this material may be considered homogeneous for butyltin and phenyltin analysis.

\section{Stability of organotins in this material}

We have been monitoring the stability of the butyltin compounds in NMIJ CRM7301-a since 2001. All three butyltins were sufficiently stable during storage for five years at $-30{ }^{\circ} \mathrm{C}$ even though CRM7301-a was not sterilized. The new CRM 7306-a contains almost the same butyltin concentrations as CRM7301-a, its sample composition was also similar, and it was sterilized with $\gamma$ ray-irradiation. Thus, the butyltins in CRM 7306-a will also be stable for at least five years. Both the phenyltin and butyltin compounds in BCR CRM646, which was pasteurized at $100{ }^{\circ} \mathrm{C}$, have been shown to be stable when the material is stored at below $-20{ }^{\circ} \mathrm{C}$ [19]. Therefore, we decided to store CRM7306-a at $-30{ }^{\circ} \mathrm{C}$ and to assess the stability of the material by measuring each organotin by ID-GC-ICP-MS over a period of 1 year, and that for CRM7301-a.

Characterization of the synthesized ${ }^{118}$ Sn-enriched organotins

Characterization of the synthesized ${ }^{118}$ Sn-enriched organotins was performed by GC-ICP-MS after mixing of each, dilution with methanol, and ethylation with $\mathrm{NaBEt}_{4}$.

Figure 2 shows the GC-ICP-MS chromatograms obtained at $\mathrm{m} / \mathrm{z} 118$ and 120 for the synthesized ${ }^{118} \mathrm{Sn}$ enriched organotins mixture. The ${ }^{118} \mathrm{Sn}$-enriched DPhT and TPhT could be synthesized individually. The molar ratio of

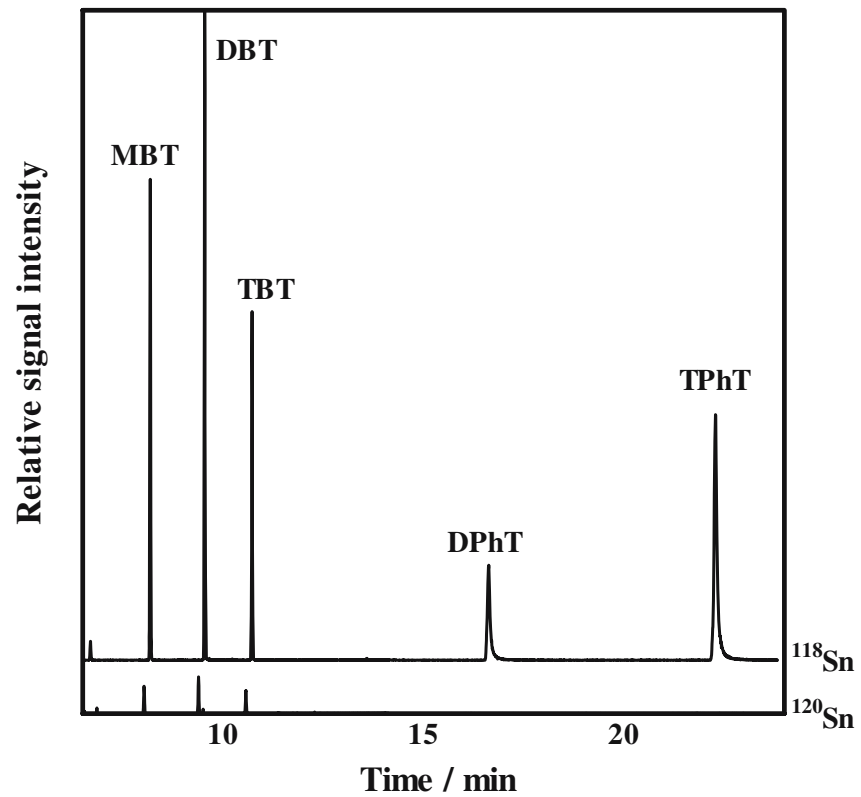

Fig. 2 GC-ICP-MS chromatograms of ${ }^{118} \mathrm{Sn}$ and ${ }^{120} \mathrm{Sn}$ obtained for the mixture of ${ }^{118} \mathrm{Sn}$-enriched organotin compounds. The chromatogram of ${ }^{118} \mathrm{Sn}$ was shifted $9 \mathrm{~s}$ and $7500 \mathrm{cps}$ for clarity

the ${ }^{118} \mathrm{Sn}-\mathrm{MBT}$, ${ }^{118} \mathrm{Sn}-\mathrm{DBT}$, and ${ }^{118} \mathrm{Sn}-\mathrm{TBT}$ in the synthesized mixture measured by $\mathrm{GC}-\mathrm{ICP}-\mathrm{MS}$ was approximately $7: 10: 5$.

Ten replicate measurements of the ethylated ${ }^{118} \mathrm{Sn}$ enriched organotin mixture by $\mathrm{GC}-\mathrm{ICP}-\mathrm{MS}$ were performed to obtain the ${ }^{120} \mathrm{Sn} /{ }^{118} \mathrm{Sn}$ ratio for each compound. The mass bias was corrected with the inrun correction method with TPrT [4]. The ${ }^{120} \mathrm{Sn} /{ }^{118} \mathrm{Sn}$ isotope ratios for ${ }^{118} \mathrm{Sn}$-butyltins were $0.055 \pm 0.001$ (mean \pm standard uncertainty, $n=10$ ), with no significant differences. The ${ }^{120} \mathrm{Sn} /{ }^{118} \mathrm{Sn}$ isotope ratios for ${ }^{118} \mathrm{Sn}$ enriched DPhT and TPhT were $0.0020 \pm 0.0002$ and $0.00020 \pm 0.0003$, respectively.

Assay for the standard solution of the organotins

To obtain a standard solution with a well-defined concentration, an assay was performed to determine the purity of the natural abundance organotin chloride reagents used to prepare the standard solutions. Details of the assay have been described elsewhere [4]. In brief, the amounts of organic and inorganic tin species impurities in the organotin chloride reagents were estimated by $\mathrm{GC}-\mathrm{ICP}-$ MS. The inorganic impurities, except for Sn, were checked by ICP-MS. The non-organotin organic impurities were also checked by GC-FID. The water content of the organotin chloride reagents were evaluated with a KarlFisher coulometric titrator (Model CA-05; Mitsubishi, Japan).

The main impurities in the organotin chloride reagents used are water and tin species. Small amounts of inorganic and organotin impurities were observed in both the ethylated MBT and ethylated DBT solutions, and signif- 


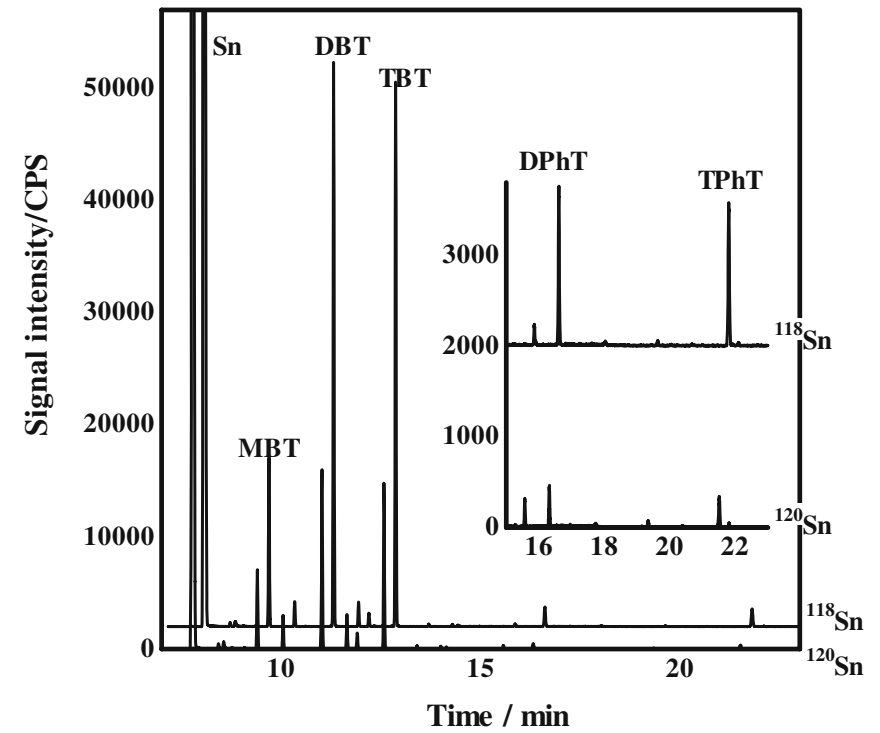

Fig. 3 GC-ICP-MS chromatograms of ${ }^{118} \mathrm{Sn}$ and ${ }^{120} \mathrm{Sn}$ obtained for the sample extract spiked with ${ }^{118}$ Sn-enriched butyltin and phenyltin compounds. The chromatogram of ${ }^{118} \mathrm{Sn}$ was shifted $7 \mathrm{~s}$ and $2000 \mathrm{cps}$ for clarity

icant amounts of several organotin impurities were observed in the ethylated TBT, ethylated TPhT, and ethylated DPhT solutions. Among the organotin impurities observed in each ethylated organotin solution, inorganic tin, MBT, DBT and tetrabutyltin (TeBT), MPhT, and DPhT were identified from their retention times in GC-ICP-MS measurement and isomers of TBT (iso-TBT) and dioctyldibutyltin (DOcDBT) were identified from the fragmention patterns obtained by GC-MS. Two organotin impurities that could not be identified from their retention time were also observed in the ethylated-MBT solution, but the amounts of those were small $(<0.03 \%)$. The purities of the organotin chloride reagent obtained were $99.4 \pm 0.2 \%$ for MBT, $99.5 \pm 0.2 \%$ for DBT, $96.8 \pm 0.2 \%$ for TBT, $98.6 \pm$ $0.2 \%$ for DPhT, and $98.8 \pm 0.2 \%$ for TPhT.

The stability of the standard solution during certification is also important. Van et al. reported that DPhT in methanol was not stable because of its disproportionation reaction, although solutions of the butyltins and TPhT were found to be stable [20]. DPhT also reacted with MBT in mixed solutions in methanol. In contrast, Arnold et al. reported that DPhT in methanol containing $0.01 \mathrm{~mol} \mathrm{~L}^{-1} \mathrm{HCl}$ was stable for six months [21]. Hence, the stability test for DPhT in methanol with and without $0.005 \mathrm{~mol} \mathrm{~L}^{-1} \mathrm{HCl}$ was performed by GC-ICP-MS after storage at $-20^{\circ} \mathrm{C}$ for one month. Redistribution of DPhT to MPhT and TPhT was observed for the methanol solution, as has been described elsewhere [20]. In contrast, no degradation or redistribution of DPhT was observed for the methanol solution containing $0.005 \mathrm{~mol} \mathrm{~L}^{-1} \mathrm{HCl}$. Plazzogna et al. reported that the redistribution reaction between monomethyltrialkyltin $\left(\mathrm{R}_{3} \mathrm{SnMe}\right)$ and dimethyltin dichloride $\left(\mathrm{Me}_{2} \mathrm{SnCl}_{2}\right)$ in methanol is occurred with dissociation of $\mathrm{Me}_{2} \mathrm{SnCl}_{2}$ [22]. They also reported that the redistribution reaction can be prevented by addition of chloride ion, as
$\mathrm{NaCl}$, which inhibits the dissociation of $\mathrm{Me}_{2} \mathrm{SnCl}_{2}$ in methanol. For DPhT in methanol the disproportionation reaction occurred with dissociation of $\mathrm{DPhTCl}_{2}$, and was prevented by addition of $\mathrm{HCl}$, which inhibited the dissociation. Consequently, the standard solution of DPhT was prepared by dissolving $\mathrm{DBTCl}_{2}$ in methanol containing $0.005 \mathrm{~mol} \mathrm{~L}^{-1} \mathrm{HCl}$ to prevent the self-disproportionation reaction.

Evaluation of the degradation of DBT and TPhT during extraction

Because SSID-MS methodology cannot compensate for non-quantitative extraction from the sample, aggressive conditions are required for solid-liquid extraction of strongly adsorbed species, for example MBT. Degradation or interconversion of species can occur under the aggressive extraction conditions [6-11]. Indeed, it has been reported that degradation of DBT occurs during microwave assisted extraction [6] and pressurized liquid extraction [7] when high-energy microwaves or high extraction temperatures are used. Degradation of TPhT during sample pretreatment has also been reported $[11,12]$. In this certification, therefore, degradation of DBT and TPhT during extraction was checked using an ${ }^{117} \mathrm{Sn}-$ enriched DBT solution obtained from the LGC, UK [15] and the single solution of the synthesized ${ }^{118} \mathrm{Sn}$-enriched TPhT. Both ${ }^{117} \mathrm{Sn}$-enriched DBT and ${ }^{118} \mathrm{Sn}$-enriched TPhT solutions were used to spike the sediment sample and then the four extraction methods were performed. After derivatization and clean-up the extract was analyzed by GCICP-MS. Because the ${ }^{118} \mathrm{Sn}$-enriched TPhT solution does not contain any phenyltins, degradation of TPhT in each

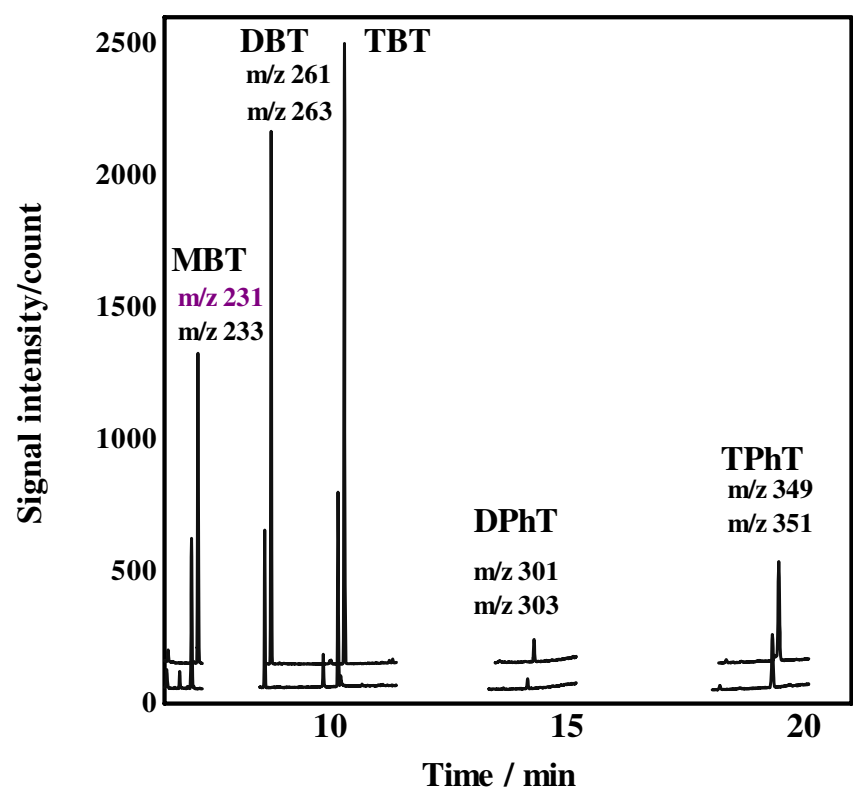

Fig. 4 GC-MS chromatograms of each measured mass obtained for the sample extract spiked with ${ }^{118} \mathrm{Sn}$-enriched butyltin and phenyltin compounds. The chromatogram for ${ }^{118} \mathrm{Sn}$ was shifted $9 \mathrm{~s}$ and $500 \mathrm{cps}$ for clarity 


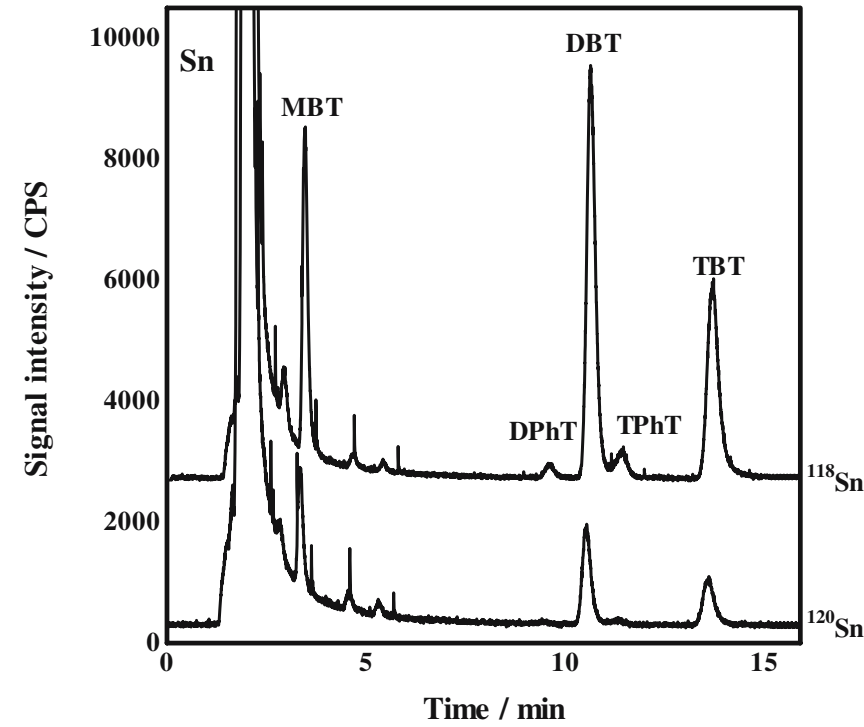

Fig. 5 LC-ICP-MS chromatograms of ${ }^{118} \mathrm{Sn}$ and ${ }^{120} \mathrm{Sn}$ obtained for the sample extract spiked with ${ }^{118} \mathrm{Sn}$-enriched butyltin and phenyltin compounds. The chromatogram of ${ }^{118} \mathrm{Sn}$ was shifted $9 \mathrm{~s}$ and $2500 \mathrm{cps}$ for clarity

extraction was evaluated from the difference between the natural abundance isotope ratio and the measured ratios of ${ }^{120} \mathrm{Sn} /{ }^{118} \mathrm{Sn}$ for DPhT. On the other hand, the ${ }^{117} \mathrm{Sn}-$ enriched DBT solution contains small amounts (ca. 0.7\%) of ${ }^{117} \mathrm{Sn}$-enriched MBT as an impurity. Therefore, the degradation of DBT in each extraction was evaluated from the difference between the natural abundance isotope ratios and the ratios of ${ }^{118} \mathrm{Sn} /{ }^{117} \mathrm{Sn}$ for MBT, corrected for ${ }^{117} \mathrm{Sn}$ enriched MBT. In this experiment, the measured ${ }^{120} \mathrm{Sn} /{ }^{118} \mathrm{Sn}$ ratios for DPhT obtained for all four extraction methods closely matched the natural abundance, and the results suggest that no significant degradation of TPhT to DPhT occurred during extraction. The degradation of DBT to MBT was also not observed in ultrasonic extraction and mechanical shaking extraction. Slight degradation of DBT to MBT was observed in microwave-assisted extraction MAE $(0.8 \%)$ and in pressurized liquid extraction PLE $(0.5 \%)$; these degradation levels were lower than those reported by Encinar $[6,7](2-3 \%$ in both extractions under their optimized conditions). These differences could be because of different extraction conditions. The sample size used in our experiments was two to four times that used by Encinar, and the concentrations of acetic acid we used were lower. In addition, tropolone was used as a chelating reagent in all the four extraction methods. Formation of the tropolone-chelating complex might prevent degradation. The slight degradation of DBT observed would not significantly affect the analytical results obtained by use of MAE and PLE because the typical uncertainty of isotope-ratio measurement by GC-ICP-MS in this study was in the range 0.5 to $1 \%$, and the relative standard deviations of the analytical results for DBT obtained by use of MAE and PLE were approximately 3\%. As is shown later, the analytical results obtained by use of the four different extraction methods were in good agreement within the range of uncertainties. These results indicate that analytical biases caused by degradation or interconversion of analytes in the extraction would be negligible in this certification.

\section{Analytical results obtained by each method}

Determination of the organotin compounds in the candidate material by the six analytical methods was performed for the certification. Figures 3, 4 to 5 show the GC-ICP-MS, GC-MS, and LC-ICP-MS chromatograms obtained for the sample extract spiked with the ${ }^{118}$ Sn-enriched organotin compounds. It is apparent from Figs. 3 and 4 that adequate sensitivity for all the organotins was obtained in GC-ICP-MS and GC-MS measurements. Adequate sensitivity for the organotin compounds, except for DPhT, was also obtained in LC-ICP-MS; the sensitivity for DPhT was insufficient to obtain a reliable isotope-ratio measurement. The relative standard deviation for DPhT in LC-ICP-MS measurement was approximately $10 \%$ in triplicate measurement, and thus variation of the analytical results obtained was quite large. Thus, the analytical results for DPhT obtained by ID-LC-ICP-MS were not adopted for the certification. The analytical results, with their uncertainties, obtained by both analytical techniques are summarized in Table 1 . The values were calculated as mass fractions (based on dry mass). The combined standard uncertainties for the analytical results obtained by each

Table 1 Analytical results obtained by use of six combinations of extraction and measurement methods

\begin{tabular}{|c|c|c|c|c|c|c|}
\hline \multirow[b]{2}{*}{ Extraction $^{\mathrm{b}}$} & \multirow[b]{2}{*}{ Measurement } & \multicolumn{5}{|c|}{ Result \pm combined standard $u^{\mathrm{a}}\left(\mu \mathrm{g} \mathrm{kg}^{-1}\right.$ as $\left.\mathrm{Sn}\right)$} \\
\hline & & TBT & DBT & MBT & $\mathrm{TPhT}$ & $\mathrm{DPhT}$ \\
\hline USE & GC-ICP-MS & $44.3 \pm 1.1$ & $51.2 \pm 0.9$ & $65.9 \pm 1.1$ & $7.8 \pm 1.4$ & $3.4 \pm 0.3$ \\
\hline USE & GC-MS & $43.8 \pm 1.7$ & $50.7 \pm 0.8$ & $66.6 \pm 1.7$ & $5.8 \pm 1.5$ & $3.9 \pm 0.4$ \\
\hline USE & LC-ICP-MS & $44.2 \pm 1.7$ & $50.7 \pm 0.8$ & $65.6 \pm 1.7$ & $7.4 \pm 1.9$ & $-^{\mathrm{c}}$ \\
\hline MAE & GC-ICP-MS & $43.8 \pm 1.5$ & $52.1 \pm 0.9$ & $68.2 \pm 1.2$ & $7.0 \pm 1.0$ & $2.9 \pm 0.1_{3}$ \\
\hline PFE & GC-ICP-MS & $42.9 \pm 1.2$ & $50.1 \pm 1.0$ & $67.1 \pm 0.8$ & $6.3 \pm 0.6$ & $3.7 \pm 0.2$ \\
\hline MWE & GC-ICP-MS & $47.0 \pm 1.3$ & $52.5 \pm 1.2$ & $67.6 \pm 0.8$ & $8.7 \pm 1.7$ & $4.4 \pm 0.8$ \\
\hline
\end{tabular}

${ }^{a}$ Calculated by use of Eqs. (1) or (2)

${ }^{b}$ USE: ultrasonic extraction, MSE: mechanical shaking extraction, PFE: pressurized fluid extraction, MAE: microwave assisted extraction ${ }^{\mathrm{c}}$ Technically invalid 
Table 2 Certified values and their uncertainties for mass fractions of organotin compounds in NMIJ CRM 7306-a

\begin{tabular}{|c|c|c|c|c|c|}
\hline & TBT & DBT & MBT & $\mathrm{TPhT}$ & $\mathrm{DPhT}$ \\
\hline Certified value (mass fraction, $\mu \mathrm{g} \mathrm{kg}^{-1}$ as $\mathrm{Sn}$ ) & 44 & 51 & 67 & 6.9 & 3.4 \\
\hline \multicolumn{6}{|l|}{ Relative standard uncertainty (\%) } \\
\hline Calibration solution $u_{\text {cal }}$ & $0.3 \%$ & $0.3 \%$ & $0.3 \%$ & $0.5 \%$ & $1.0 \%$ \\
\hline Analytical results $u_{\text {anal }}$ & $1.3 \%$ & $0.7 \%$ & $0.7 \%$ & $7.0 \%$ & $3.3 \%$ \\
\hline Between method $u_{\text {method }}$ & $1.6 \%$ & $1.0 \%$ & $0.2 \%$ & - & - \\
\hline In-homogeneity $s_{\mathrm{bb}}$ or $u_{\mathrm{bb}}$ & $1.9 \%$ & $1.9 \%$ & $2.0 \%$ & $5.8 \%$ & $7.1 \%$ \\
\hline \multicolumn{6}{|l|}{ Combined uncertainty } \\
\hline Relative (\%) & $2.8 \%$ & $2.3 \%$ & $2.1 \%$ & $9.1 \%$ & $7.9 \%$ \\
\hline Absolute $\left(\mu \mathrm{g} \mathrm{kg}^{-1}\right.$ as $\left.\mathrm{Sn}\right)$ & 1.3 & 1.2 & 1.4 & 0.6 & 0.3 \\
\hline Expanded uncertainty $U(k=2)$ (mass fraction, $\mu \mathrm{g} \mathrm{kg}^{-1}$ as $\mathrm{Sn}$ ) & 3 & 2 & 3 & 1.3 & 0.5 \\
\hline
\end{tabular}

method were calculated by use of Eqs. (1) or (2). The uncertainties related to the standard solutions (uncertainty of $P$ and $D$ in Eqs. (1) and (2)) were not combined into $u_{\mathrm{c}}$ because the same reagents were used for both analyses. They were combined later when the uncertainties in the certified values were calculated.

The analytical results obtained by use of the six methods were in good agreement within the range of their uncertainties; this agreement may indicate there were no significant analytical biases between measurement and extraction techniques for all the analytes. Therefore, all the analytical results obtained were treated equally for calculation and evaluation of the certified values and their uncertainties.

\section{Establishing certified values}

The analytical results in Table 1 were combined to provide the certified values listed in Table 2. Certified values are the weighted means of these results from the six methods, where $1 / u_{i}\left(u_{i}\right.$ is the uncertainty of each result) was used as a weight. Certified values are available for the concentrations of MBT, DBT, TBT, DPhT, and TPhT, as tin.

\section{Uncertainty of the certified values}

The uncertainties of the certified values included the combined effects of method imprecision, possible bias effects among methods, material inhomogeneity, and stability. Components of the uncertainty of each certified value are listed in Table 2. Because the same reagents for calibration solutions were used for all the measurements, uncertainty of the calibration solutions $\left(u_{\mathrm{cal}}\right)$ was combined with the uncertainty of the certified values separately from the uncertainty of the analytical results, as is described above. The property value was the weighted mean of the two results, and so the combined uncertainty of each analytical result ( $\left.u_{\text {anal }}\right)$ was given by Eq. (5):

$u_{\mathrm{anal}}(x)=\sqrt{\sum_{i} w_{i}^{2} u^{2}\left(x_{i}\right)}$ where $x_{i}$ are the results obtained by use of the six methods and $w_{i}$ are their weights. The between-method variance $\left(u_{\text {method }}\right)$ was calculated from ANOVA on the data from the three techniques. The uncertainties derived from material inhomogeneity $\left(u_{\mathrm{bb}}\right)$ were the estimates in the homogeneity study. As is described above, the butyltin and phenyltin compounds would be stable at lest five years at our storage temperature $\left(-30^{\circ} \mathrm{C}\right)$; we did not include the uncertainty from stability. The expanded uncertainty in each certified value is equal to $U=k u_{\mathrm{c}}$, where $u_{\mathrm{c}}$ is the combined standard uncertainty, with coverage factor $k=2$, corresponding to $95 \%$ confidence intervals.

\section{Comparison with other CRMs}

The certified values for CRM7301-a and the CRM7306-a are summarized in Table 3. In comparison with the CRM7301-a, the uncertainties of the certified values for DBT and MBT in CRM7306-a are significantly smaller, despite the similar concentrations of the compounds. The main contributors to the uncertainty in the certified values for DBT and MBT in CRM7301-a were $u_{\mathrm{bb}}$ and $u_{\text {anal }}$ [3]. The homogeneity of CRM 7306-a was improved by remixing after X-ray sterilization, and the reliability of the analytical methods used for certification was also better than those used for CRM7301-a. Thus, these improvements may lead to the small uncertainties.

Most recently, several sediment reference materials for butyltin analysis certified by use of SSID-MS have been issued by the National Research Council Canada (NRCC, Canada). The certified values of the CRMs are also

Table 3 Certified values for butyltin and phenyltin in MNIJ7306-a and other CRMs certified by use of SSIDMS (unit: $\mu \mathrm{g} \mathrm{kg}^{-1}$ as $\mathrm{Sn}$ )

\begin{tabular}{llllll}
\hline & TBT & DBT & MBT & TPhT & DPhT \\
\hline CRM7306-a & $44 \pm 3$ & $51 \pm 2$ & $67 \pm 3$ & $6.9 \pm 1.2$ & $3.4 \pm 0.5$ \\
CRM7301-a & $44 \pm 4$ & $56 \pm 6$ & $56 \pm 6$ & & \\
HIPA-1 & $78 \pm 9$ & & & & \\
SOPH-1 & $125 \pm 7$ & $174 \pm 9$ & & & \\
PACS-2 & $890 \pm 105$ & $1047 \pm 64$ & & & \\
\hline
\end{tabular}


summarized in Table 3. HIPA-1 and SOPH-1 were certified, and PACS-2 was recertified by NRCC. Their certified and re-certified values were based on results from the Comite Consultatif pour la Quantite de Matiere (CCQM) comparisons [23-25], in which we participated. These CRMs have certified values for DBT and TBT only, and their concentrations are higher than those in CRM7301-a and CRM7306-a.

The BCR 646 freshwater sediment for butyltins and phenyltins are also available from the European Commission Joint Research Centre (IRMM, Belgium), although SSID-MS was not used for the certification. The certified concentrations for BCR CRM646 are higher than those for CRM7306-a.

In Japan, typical butyltin and phenyltin concentrations observed in environmental monitoring are not as high as they used to be. Reported TBT concentrations in sediment were below $80 \mu \mathrm{g} \mathrm{kg}^{-1}$, as $\mathrm{Sn}$, in environmental monitoring by the Ministry of the Environment Japan in 1998 [26]. By using these CRMs properly, a wide concentration range can be covered in routine laboratories.

Acknowledgements The authors thank LGC Ltd. (Teddington, UK) for providing the ${ }^{117} \mathrm{Sn}$-enriched DBT solution. Sample collection and preparation were performed by Environmental Technology Service Co., Ltd. This research was supported by the millennium research project of the Japanese government.

\section{References}

1. Hoch M (2001) Appl Geochem 16:719-743

2. Quevauviller Ph, Donard OFX (2001) Trends in Anal Chem 20:600-613

3. Inagaki $\mathrm{K}$, Takatsu $\mathrm{A}$, Watanabe $\mathrm{T}$, Kuroiwa $\mathrm{T}$, Aoyagi $\mathrm{Y}$, Okamoto K (2004) Anal Bioanal Chem 378:1265-1270

4. Inagaki $\mathrm{K}$, Takatsu $\mathrm{A}$, Watanabe $\mathrm{T}$, Aoyagi $\mathrm{Y}$, Okamoto $\mathrm{K}$ (2003) Analyst 128:265-272

5. Yang L, Bancon-Montigny C, Mester Z, Sturgeon RE, Willie SN, BoykoVJ (2003) Anal Bioanal Chem 376:85-91

6. Encinar JR, Rodriguez-Gonzalez P, Alonso JIG, Sanz-Medel A (2002) Anal Chem 74:270-281
7. Encinar JR, Rodriguez-Gonzalez P, Fernandez JR, Alonso JIG, Diez S, Bayona JM, Sanz-Medel A (2002) Anal Chem 74:5237-5242

8. Rodriguez-Gonzalez P, Encinar JR, Alonso JIG, Sanz-Medel A (2003) Analyst 128:447-452

9. Rodriguez-Gonzalez P, Alonso JIG, Sanz-Medel A (2005) J Anal At Spectrom 20:1076-1084

10. Kumar SJ, Tesfalidet S, Snell J, Frech W (2003) J Anal At Spectrom 18:714-719

11. Kumar SJ, Tesfalidet S, Snell J, Van DN, Frech W (2004) J Anal At Spectrom 19:368-372

12. Abalos M, Bayona JM, Quevauviller P (1998) Appl Organomet Chem 12:541-549

13. Chau YK, Yang F, Maguire RJ (1996) Anal Chim Acta 320:165-169

14. Ceulemans M, Adams FC (1995) Anal Chim Acta 317:161-170

15. Sutton PG, Harrington CF, Fairman B, Evans EH, Ebdon L, Catterick T (2000) Appl Organomet Chem 14:691-700

16. Henrion A (1994) Fresenius J Anal Chem 350:657-658

17. Ellison SLR, Rosslein M, Williams A (2000) EURACHEM/ CITAC guide, quantifying uncertainty in analytical measurement. 2nd edn, pp 89-96

18. Linsinger TP, Pauwels J, van der Veen AMH, Schimmel H, Lamberty A (2001) Accred Qual Assur 6:20-25

19. Quevauviller Ph, Ariese F (2001) Trends in Anal Chem 20:207-218

20. Van DN, Lindberg R, Frech W (2005) J Anal At Spectrom $20: 266-272$

21. Arnord CG, Berg M, Müller SR, Dommann U, Schwarzenbach RP (1998) Anal Chem 70:3094-3101

22. Piazzogna G, Bresadola bS, Tagliavini G (1967) Inorg Chim Acta 2:333-336

23. Sturgeon RE, Wahlen R, Brandsch T, Fairman B, Wolf-Briche C, Garcia Alonso JI, Rodriguez González P, Ruiz Encinar J, Sanz-Medel A, Inagaki K, Takatsu A, Lalere B, Monperrus M, Zuloaga O, Krupp E, Amouroux D, Donard OFX, Schimmel H, Sejerøe-Olsen B, Konieczka P, Schultze P, Taylor P, Hearn R, Mackay L, Myors R, Win T, Liebich A, Philipp R, Yang L, Willie S (2003) Anal Bioanal Chem 376:780-787

24. Wolff Briche CSJ, Wahlen R, Sturgeon RE (2006) Metrologia 43:Tech. Suppl., 08001

25. Wolff Briche CSJ, Wahlen R, Sturgeon RE (2006) Metrologia 43:Tech. Suppl., 08002

26. Environmental health and safety division in ministry of the environment Japan (ed) (2000) Summary of the survey results for organotin compounds (Fiscal Year 1998) in "Report on environmental survey and wildlife monitoring of chemicals in F. Y. 1998" 